\title{
Predicting functional impairment in brain tumor surgery: the Big Five and the Milan Complexity Scale
}

\author{
*Paolo Ferroli, MD, ${ }^{1}$ Morgan Broggi, MD, PhD, ${ }^{1}$ Silvia Schiavolin, PsyD, ${ }^{2}$ \\ Francesco Acerbi, MD, PhD, ${ }^{1}$ Valentina Bettamio, MD, ${ }^{3}$ Dario Caldiroli, MD, ${ }^{1}$ Alberto Cusin, MD, ${ }^{1}$ \\ Emanuele La Corte, MD, ${ }^{1}$ Matilde Leonardi, MD, ${ }^{2}$ Alberto Raggi, PsyD, ${ }^{2}$ Marco Schiariti, MD, ${ }^{1}$ \\ Sergio Visintini, MD, ${ }^{1}$ Angelo Franzini, MD, ${ }^{1}$ and Giovanni Broggi, MD ${ }^{1}$
}

Departments of ${ }^{1}$ Neurosurgery, ${ }^{2}$ Neurology, Public Health, and Disability Unit-Scientific Directorate, and ${ }^{3}$ Medical Directorate, Fondazione IRCCS Istituto Neurologico Carlo Besta, Milano, Italy

OBJECT The Milan Complexity Scale-a new practical grading scale designed to estimate the risk of neurological clinical worsening after performing surgery for tumor removal-is presented.

METHODS A retrospective study was conducted on all elective consecutive surgical procedures for tumor resection between January 2012 and December 2014 at the Second Division of Neurosurgery at Fondazione IRCCS Istituto Neurologico Carlo Besta of Milan. A prospective database dedicated to reporting complications and all clinical and radiological data was retrospectively reviewed. The Karnofsky Performance Scale (KPS) was used to classify each patient's health status. Complications were divided into major and minor and recorded based on etiology and required treatment. A logistic regression model was used to identify possible predictors of clinical worsening after surgery in terms of changes between the preoperative and discharge KPS scores. Statistically significant predictors were rated based on their odds ratios in order to build an ad hoc complexity scale. For each patient, a corresponding total score was calculated, and ANOVA was performed to compare the mean total scores between the improved/unchanged and worsened patients. Relative risk (RR) and chi-square statistics were employed to provide the risk of worsening after surgery for each total score.

RESULTS The case series was composed of 746 patients (53.2\% female; mean age $51.3 \pm 17.1)$. The most common tumors were meningiomas $(28.6 \%)$ and glioblastomas $(24.1 \%)$. The mortality rate was $0.94 \%$, the major complication rate was $9.1 \%$, and the minor complication rate was $32.6 \%$. Of 746 patients, $523(70.1 \%)$ patients improved or remained unchanged, and 223 (29.9\%) patients worsened. The following factors were found to be statistically significant predictors of the change in KPS scores: tumor size larger than $4 \mathrm{~cm}$, cranial nerve manipulation, major brain vessel manipulation, posterior fossa location, and eloquent area involvement (Nagelkerke $R^{2}=0.286$ ). A grading scale was obtained with scores ranging between 0 and 8 . Worsened patients showed mean total scores that were significantly higher than the improved/unchanged scores $(3.24 \pm 1.55$ vs $1.47 \pm 1.58$; $p<0.001)$. Finally, a grid was developed to show the risk of worsening after surgery for each total score: scores higher than 3 are suggestive of worse clinical outcome.

CONCLUSIONS Through the evaluation of the 5 aforementioned parameters - the Big Five-the Milan Complexity Scale enables neurosurgeons to estimate the risk of a negative clinical course after brain tumor surgery and share these data with the patient. Furthermore, the Milan Complexity Scale could be used for research and educational purposes and better health system management.

http://thejns.org/doi/abs/10.3171/2015.9.FOCUS15339

KEY WORDS brain tumor; complication; outcome; grading system; Karnofsky Performance Scale; craniotomy

ABBREVIATIONS FINCB = Fondazione IRCCS Istituto Neurologico Carlo Besta; GTR = gross-total resection; KPS = Karnofsky Performance Scale; OR = odds ratio; RR = relative risk; STR = subtotal resection.

SUBMITTED July 30, 2015. ACCEPTED September 28, 2015.

INCLUDE WHEN CITING DOI: 10.3171/2015.9.FOCUS15339.

* Drs. Ferroli and M. Broggi contributed equally to this work. 
$\mathrm{R}$ EPORTING complications, quality, and outcome assessments in neurosurgery have gained paramount importance, not only for their therapeutic values but also as ways to evaluate health system efficiency and efficacy, especially during periods of global economic crisis and limited resources. ${ }^{16,19,28}$ This is not a new concept. Around 100 years ago, Harvey Cushing, one of the pioneers of neurosurgery, thoroughly documented his patients' complications. ${ }^{24}$ Despite this, today's administrators allocate resources according to indicators whose meanings are not evenly well-defined and, therefore, potentially misleading. ${ }^{18,39}$

The issue of how to define the negative results of surgical procedures were initially a matter of debate in the field of general surgery and recently in the neurosurgical community. $17,18,23,31,35$ More than 20 years ago, general surgeons defined a complication as any morbid event occurring "as a result of the procedure, during the performance or recovery from the procedure, which causes deviations from the ideal course and tends to impair or delay complete recovery and induces changes in the management of the patient." 10 The same authors brought up another pivotal concept: the complexity of surgery, which arises from the preoperative conditions and is defined as the risk to incur in postoperative complications and negative outcomes. Indeed, in order to assess the preoperative risk factors of patients, a scale that takes into account the patient's age and concomitant morbidities was proposed..$^{10}$ Later on, several variations of the classification were proposed by different authors, ${ }^{15,20,21,39}$ but until recently a definition of surgical complication that was accepted by the entire scientific community was still lacking. The issue regarding what is a complication and how it should be classified has also been an object of debate for many years in neurosurgery. ${ }^{2,5-7,22,23,26,38}$

Nevertheless, patients do not care how complications and outcomes in neurosurgery are defined. Their main question would be: "Given that I have to undergo surgery, where do I have to go to have a greater possibility of getting rid of my tumor without negative outcomes?" It has been demonstrated that the hospital and surgeon case volume have an impact on outcomes across a variety of subspecialties, including neurosurgery, ${ }^{3,4,12,13,33}$ and these volumes' relationships with mortality and discharge disposition after biopsy or resection of primary brain tumors have also been shown. ${ }^{3,29}$ Nevertheless, to answer that particular question, estimating the specific risk related to a single neurosurgical procedure is critical. In brief, to answer the question, "Where do I have to go?", the only existing viable way until now has been to address performance in terms of a single institution's case volume. However, case volume is an indirect index that does not take into account the complexity of each case and does not control for selection biases; e.g., the decision of whether or not to intervene in difficult and complex cases, as correctly noted by Clark and Spetzler. ${ }^{9}$ However, neurosurgery is such a vast discipline that the entire neurosurgical patient population would be too heterogeneous to build up a specific and effective risk scale. Consequently, it would be ideal to preoperatively define the risk-related factors and complexity of surgery for each class of neurosurgical procedures, as
Spetzler and Martin did in 1986 for arteriovenous cerebral malformations ${ }^{36}$ Hence, we thought that focusing on brain tumors would render a wide enough, representative, and fairly homogeneous sample.

This study was therefore designed to identify the impact of some important preoperative factors on the outcomes of brain tumor surgery, with the aim of building a new, easy, and practical grading scale capable of predicting the risk of clinical worsening after performing a craniotomy or endoscopic endonasal approach for tumor removal. The scale should be able to grade tumors on the basis of predictors of surgical complexity and outcome.

\section{Methods}

\section{Study Type and Inclusion Criteria}

This retrospective analysis was conducted on all elective brain neuro-oncological surgical procedures performed at the Second Division of Neurosurgery, Fondazione IRCCS Istituto Neurologico Carlo Besta (FINCB) of Milan, Italy between January 2012 and December 2014. The records of patients of all ages who received a diagnosis of a possible brain tumor of any type and underwent surgery aimed at a maximal safe tumor resection, e.g., craniotomy for tumor removal or endoscopic endonasal resection, were reviewed. Cases referred for craniotomy for open biopsy, stereotactic/frameless biopsy, or endoscopic endonasal biopsy were excluded. The decision-making approach used at our institution follows a maximal safe resection philosophy, i.e., to tailor the extent of resection based on the tumor's features, such as location and size, and the patient's features, such as age and comorbidities, in order to remove as much tumor as possible without impairing the patient's general health status. For tumors located in eloquent areas, extended neurophysiological monitoring and, in selected cases, the use of awake craniotomy, is proposed to map the cortical and subcortical eloquent areas and reduce the incidence of postoperative deficits. ${ }^{11}$

Sociodemographic and neurosurgical data were prospectively collected, recorded, and retrospectively revised by a dedicated neurosurgeon through the Neurosurgical Complications Protocol and Database Besta-NSC.17 The Karnofsky Performance Scale (KPS) was used to evaluate health status before surgery and at discharge using physical examination. KPS was chosen since, as shown in a recent review, ${ }^{30}$ it has the strongest support in the literature for predicting surgery-related outcomes and was shown to be effective in predicting early ( $\leq 30$-day) morbidity in intracranial tumor patients. Besides preoperative and discharge KPS scores, neurosurgical complications were recorded as major or minor based on the etiology and treatment required to address them ${ }^{23}$ in order to describe the clinical course after surgery. Major complications were considered as follows: new or worse impaired neurological function (e.g., hemiparesis, hemianopia), cranial nerve palsies, stroke, sepsis, "major" re-craniotomy (e.g., blood clot/subdural/extradural hematoma removal, decompressive craniectomy for brain swelling, surgical CSF leak repair), and life-threatening medical complications (e.g., heart complications, pulmonary embolism). Minor complications were considered as follows: wound infec- 
tions, postoperative meningitis, subgaleal fluid collections, subjective neurological deficits (e.g., visual disturbances, confusion), postoperative fever or minor infections (e.g., urinary tract infections), and "minor" re-craniotomy (e.g., wound revision, external ventricular drainage, ventriculoperitoneal shunt, external spinal drainage for CSF leak repair). Etiologic classes were as follows: traumatic (i.e., directly related to the surgical trauma/manipulation of a specific structure), CSF-related (i.e., leaks, hydrocephalus), infectious, hemorrhagic, ischemic, epileptic, general (extra-central nervous system), or medicine-related (i.e., lung or urological infection, cardiac arrhythmias). The study was approved by the ethics committee of FINCB, and all patient signed an informed consent form.

\section{Statistical Analyses}

Descriptive statistics, frequencies, and percentages were used to report the sociodemographic, clinical, and neurosurgical variables. The following statistical analyses were performed to build the grading scale.

First, a logistic regression model was built to investigate the strength of the relationship between the change in health status after surgery and sociodemographic and neurosurgical factors. An outcome was defined as the difference in the KPS scores before surgery and discharge and was dichotomized into improved/unchanged or worsened. Candidate factors were as follows: age $(0-44,45-60$, or $\geq$ 61 years), tumor size $(0-4 \mathrm{~cm}$ or $\geq 4.1 \mathrm{~cm})$, surgery in an eloquent area (yes or no), manipulation of the major brain vessels (yes or no), cranial nerve manipulation (yes or no), surgery on the brainstem (yes or no), and surgery in the posterior fossa (yes or no). All factors were evaluated on preoperative radiological images (MR, CT, digital angiography, angio-CT, or angio-MR). Specifically, surgery in an eloquent area was considered as follows: motor, sensory, language or visual areas, hypothalamus, thalamus, internal capsule, brainstem, and pineal region.

We used the odds ratio (OR) and Nagelkerke $\mathrm{R}^{2}$ to evaluate the goodness of fit of the model. The goodness of fit of the final and baseline models was compared by computing the difference in their -2log likelihoods and chisquare statistics. The composition of the groups in reference to tumor size was carried out using cluster analysis in order to maximize the differences between the 2 groups.

Second, we rated the significant predictors after rounding their ORs to the closest even number. For each patient, a corresponding total score was calculated based on the sum of all predictors' scores. The ANOVA test was performed to compare the mean Milan Complexity Scale scores between the worsened and improved/unchanged groups.

To test the performance of our scale, we ran 2 different subsample analyses. First, we took into account the cases with gross-total resection (GTR) and subtotal resection (STR). In these 2 samples, we compared the mean Milan Complexity Scale scores between the worsened and improved/unchanged groups using ANOVA. Second, we used the same procedure by defining subsamples on the basis of their histological findings, and divided cases according to the presence of extraaxial or intraaxial tumors. To have the most homogeneous samples possible, we lim- ited this second analysis to cases with meningioma and glioma.

Finally, the risk of impairment after surgery for each total score of the scale was calculated using relative risk (RR) and chi-square statistics. Scores representing fewer than 10 patients were grouped together.

Data were analyzed using SPSS (version 18.0, IBM).

\section{Results}

A total of 746 brain neurooncological procedures were performed during the study period. The series was composed of 397 (53.2\%) female and 349 (46.8\%) male patients with a mean age of $51.3 \pm 17.1$ years and a range between 1 and 87 years. The procedures performed included 661 craniotomies for tumor removal $(88.6 \%)$ and 85 endoscopic endonasal tumor resections (11.4\%). The most frequent tumors were meningiomas $(28.6 \%)$ and glioblastomas $(24.1 \%)$, followed by adenomas $(8.4 \%)$, anaplastic astrocytomas (8\%), low-grade gliomas (7.2\%), and metastases (6.2\%). Table 1 summarizes the sociodemographic, clinical, and neurosurgical data.

The mortality rate was $0.94 \%$ in this series. Complications of any kind were recorded in $311(41.7 \%)$ patients: of these, 68 were major complications $(9.1 \%$ of all procedures) and $243(32.6 \%)$ were minor complications (Table 2). The 311 complications classified on the basis of the required treatment ${ }^{23}$ were distributed as follows (Landriel Ibañez classification): 216 Grade I complications, which did not require invasive treatment $(69.4 \%$ of cases with complications); 69 Grade II complications, which required

TABLE 1. Sociodemographic, clinical, and neurosurgical data (n $=746$ )

\begin{tabular}{lc}
\hline \multicolumn{1}{c}{ Variable } & Value $^{*}$ \\
\hline Sex & $349(46.8)$ \\
\hline Male & $397(53.2)$ \\
\hline Female & \\
\hline Age (yrs) & $51.3 \pm 17.1$ \\
\hline Mean \pm SD & $1-87$ \\
\hline Range & $213(28.6)$ \\
\hline Histology & $180(24.1)$ \\
\hline Meningioma & $63(8.4)$ \\
\hline Glioblastoma & $60(8.0)$ \\
\hline Adenoma & $54(7.2)$ \\
\hline Anaplastic astrocytoma & $46(6.2)$ \\
\hline Low-grade glioma & $35(4.7)$ \\
\hline Metastasis & $21(2.8)$ \\
\hline Schwannoma & $11(1.5)$ \\
\hline Dermoid \& epidermoid cysts & $10(1.3)$ \\
\hline Chordoma & $53(7.1)$ \\
\hline Craniopharyngioma & $661(88.6)$ \\
\hline Other & $85(11.4)$ \\
\hline Type of surgery &
\end{tabular}

* Values are number of patients (\%) unless indicated otherwise. 
TABLE 2. Outcomes and complications

\begin{tabular}{cc}
\hline \multicolumn{1}{c}{ Variables } & No. of Patients $(\%)$ \\
\hline Change in KPS score after surgery & \\
\hline Worsened & $223(29.9)$ \\
\hline Improved/unchanged & $523(70.1)$ \\
\hline Mortality & $7(0.94)$ \\
\hline Neurosurgical complications & $435(58.3)$ \\
\hline No complications & $311(41.7)$ \\
\hline Complications & $68(9.1)$ \\
\hline Major & $243(32.6)$ \\
\hline Minor
\end{tabular}

invasive treatment (22.2\%); 19 Grade III complications, which required management in the intensive care unit (6.1\%); and 7 Grade IV complications, i.e., patient death (2.3\% of all complications). The same 311 complications were classified on the basis of the etiological category, which considered that a single complication may have more than 1 etiology. The most frequent causes of complications were trauma (53.4\%), CSF-related causes (13.8\%), and infection (11.6\%) (Table 3). Regarding the change in KPS scores, 523 (70.1\%) patients improved or remained unchanged after surgery (Table 2). Table 4 presents the candidate predictors of outcome.

The results of logistic regression developed using the changes in KPS scores are shown in Table 5. The variable "surgery in brainstem" was excluded from the analysis because it was collinear with the variable "surgery in eloquent area." Taken together, the variables entered into the model indicated a moderate fit (Nagelkerke $\mathrm{R}^{2}=0.286$ ). All variables referred to the tumor's features were associated with an increased odds of clinical worsening, while patient age did not demonstrate any statistical association. The contribution of these neurosurgical predictors was significantly informative since the $-2 \log$ likelihood difference was large and significant (difference $=167.8$; $\mathrm{df}=7$; $\mathrm{p}<0.001)$.

We built our complexity scale-the Milan Complexity Scale-to rate each significant predictor on the basis of its OR. A grading scale was obtained, with scores ranging between 0 and 16, but we decided to reduce it to a scale of 0 to 8 in order to make it simpler and maintain the same ratio between scores. Higher scores indicate more complex clinical situations. The Milan Complexity Scale is shown in Table 6. The scores of this scale can result from 32 different combinations of the factors that have been considered.

The ANOVA test showed that the worsened group had a mean total score that was significantly higher than the improved/unchanged group $(3.24 \pm 1.55$ vs $1.47 \pm 1.58$; $\mathrm{p}<0.001)$. This result confirmed that high Milan Complexity Scale scores correspond to worse clinical situations after surgery. In this series, GTR was carried out in $76.3 \%$ of the cases, STR (>90\%) in an additional $17.0 \%$ of cases, and the remaining $6.7 \%$ were partial resections: therefore, $93.3 \%$ of patients had more than $90 \%$ of the tumor removed. The corresponding scores of the Milan Complexity Scale were $1.80 \pm 1.67$ for GTR, $2.63 \pm 1.91$
TABLE 3. Complications classified on the basis of required treatment and etiology

\begin{tabular}{cc}
\hline \multicolumn{1}{c}{ Classification of Complications } & No. of Patients (\%) \\
\hline Required treatment ${ }^{*}$ & $216(69.4)$ \\
\hline Grade I & 120 \\
\hline Grade la & 96 \\
\hline Grade Ib & $69(22.2)$ \\
\hline Grade II & 19 \\
\hline Grade Ila & 50 \\
\hline Grade IIb & $19(6.1)$ \\
\hline Grade III & 16 \\
\hline Grade IIla & 3 \\
\hline Grade IIIb & $7(2.3)$ \\
\hline Grade IV & $166(53.4)$ \\
\hline Etiological categories $\dagger$ & $43(13.8)$ \\
\hline Traumatic & $36(11.6)$ \\
\hline CSF related & $26(8.4)$ \\
\hline Septic & $20(6.4)$ \\
\hline Ischemia & $19(6.1)$ \\
\hline Hemorrhagic & $12(3.9)$ \\
\hline General medicine (extra-CNS) complications & $7(2.2)$ \\
\hline Epilepsy & \\
\hline Other & \\
\hline
\end{tabular}

* Classified according to the Landriel Ibañez classification: Grade I, any nonlife-threatening deviation from normal postoperative course that did not require invasive treatment; Grade la, complication not requiring drug treatment; Grade $\mathrm{Ib}$, complication requiring drug treatment; Grade II, complication requiring invasive treatment such as surgical, endoscopic, or endovascular interventions: Grade Ila, complication requiring intervention without general anesthesia; Grade Ilb, complication requiring intervention with general anesthesia; Grade III, life-threatening complications requiring management in the intensive care unit; Grade IIla, complication involving single organ failure; Grade IIllb, complication involving multiple organ failure; and Grade IV, complication resulting in death.

$\dagger$ The number of complications based on the etiological category was higher than those based on the Landriel Ibañez classification due to the fact that a complication may have multiple etiologies.

for STR, and $2.72 \pm 1.98$ for partial tumor removal. The results of ANOVA were significant $(p<0.001)$ : Bonferroni post hoc analysis showed that patients with GTR also had a lower score compared with patients with STR or partial resection, while the scores of patients with STR or partial resection were not different.

When the 2 subsamples of patients with GTR $(n=569)$ and STR $(\mathrm{n}=177)$ were analyzed separately, our results show that the Milan Complexity Scale demonstrated good performance: among GTR cases, the worsened group had a mean total score that was significantly higher than the improved/unchanged group $(2.99 \pm 1.42$ vs $1.38 \pm 1.54$; $\mathrm{p}<0.001$ ); among STR cases, the worsened group had a mean total score that was significantly higher than the improved/unchanged group $(3.72 \pm 1.70$ vs $1.85 \pm 1.68$; $\mathrm{p}$ $<0.001)$.

When the 2 subsamples of patients with glioma $(\mathrm{n}=$ 294) and meningioma $(\mathrm{n}=213)$ were analyzed separately, our results show that the Milan Complexity Scale demonstrated good performance. Among glioma cases, the wors- 
TABLE 4. Descriptive statistics of surgical predictors

\begin{tabular}{cc}
\hline \multicolumn{1}{c}{ Variable } & No. of Patients $(\%)$ \\
\hline Tumor size & \\
\hline $0-4 \mathrm{~cm}$ & $521(69.8)$ \\
\hline$\geq 4.1 \mathrm{~cm}$ & $225(30.2)$ \\
\hline Surgery in eloquent area & $490(65.7)$ \\
\hline No & $216(28.9)$ \\
\hline Yes, brain eloquent area & $40(5.4)$ \\
\hline Yes, brainstem & $137(18.4)$ \\
\hline Surgery in posterior fossa & $609(81.6)$ \\
\hline Yes & $92(12.3)$ \\
\hline No & $654(87.7)$ \\
\hline Major brain vessel manipulation & \\
\hline Yes & $135(18.1)$ \\
\hline No & $611(81.9)$ \\
\hline Cranial nerve manipulation
\end{tabular}

ened group had a mean total score that was significantly higher than the improved/unchanged group $(3.17 \pm 1.54$ vs $1.67 \pm 1.60 ; p<0.001)$. Among meningioma cases, the worsened group had a mean total score that was significantly higher than the improved/unchanged group (3.39 \pm 1.77 vs $1.30 \pm 1.60 ; \mathrm{p}<0.001)$.

Finally, the RR analyses allowed us to develop a grid
TABLE 6. Milan Complexity Scale

\begin{tabular}{cc}
\hline \multicolumn{1}{c}{ Variable } & Score \\
\hline Major brain vessel manipulation & 0 \\
\hline No & 1 \\
\hline Pes & 0 \\
\hline Posterior fossa & 1 \\
\hline No & \\
\hline Yes & 0 \\
\hline Cranial nerve manipulation & 2 \\
\hline No & 0 \\
\hline Yes & 3 \\
\hline Eloquent area & 0 \\
\hline No & 1 \\
\hline Yes & $0-8$ \\
\hline Tumor size &
\end{tabular}

showing the risk of clinical impairment after surgery for each total score of the scale. Scores higher than 6 were merged. As shown in Table 7, scores between 0 and 2 are indicative of no association with worsening: therefore, these patients are likely to improve or at least be stable following surgery. On the contrary, scores higher than 3 are suggestive of an increased risk of worsening.

TABLE 5. Logistic regression predicting worsening KPS and associations between clinical outcomes and sociodemographic and neurosurgical variables*

\begin{tabular}{|c|c|c|c|}
\hline Factor & B Value (SE) & OR $(95 \% \mathrm{Cl})$ & p Value \\
\hline Constant & $-2.88(0.40)$ & & $<0.001$ \\
\hline \multicolumn{4}{|c|}{ Surgery in eloquent area (including brainstem) } \\
\hline No (reference) & - & - & - \\
\hline Yes & $1.93(0.20)$ & $6.71(4.65-10.28)$ & $<0.001$ \\
\hline \multicolumn{4}{|c|}{ Major brain vessels manipulation } \\
\hline No (reference) & - & - & - \\
\hline Yes & $0.53(0.26)$ & $1.69(1.02-2.83)$ & 0.042 \\
\hline \multicolumn{4}{|l|}{ Cranial nerve manipulation } \\
\hline No (reference) & - & - & - \\
\hline Yes & $1.47(0.25)$ & $4.34(2.62-7.17)$ & $<0.001$ \\
\hline \multicolumn{4}{|l|}{ Tumor size } \\
\hline 0-4 cm (reference) & - & - & - \\
\hline$\geq 4.1 \mathrm{~cm}$ & $0.67(0.20)$ & $1.95(1.33-2.86)$ & 0.001 \\
\hline \multicolumn{4}{|l|}{ Age } \\
\hline 0-44 yrs (reference) & - & - & - \\
\hline $45-60$ yrs & $-0.15(0.22)$ & $0.86(0.56-1.32)$ & 0.490 \\
\hline$\geq 61 \mathrm{yrs}$ & $0.11(0.23)$ & $1.12(0.71-1.76)$ & 0.626 \\
\hline \multicolumn{4}{|l|}{ Surgery in posterior fossa } \\
\hline No (reference) & - & - & - \\
\hline Yes & $0.70(0.24)$ & $2.02(1.26-3.24)$ & 0.007 \\
\hline
\end{tabular}

* Model based on intercept only $-2 \log$ likelihood $=358.7$; final model $-2 \log$ likelihood $=190.9$; chi-squared $=167.8, \mathrm{df}=7, p<0.001$. Nagelkerke pseudo- $R^{2}=0.286$. 
TABLE 7. Relative risk analysis

\begin{tabular}{cccc}
\hline $\begin{array}{c}\text { Milan Com- } \\
\text { plexity Scale } \\
\text { Score }\end{array}$ & $\begin{array}{c}\text { Score } \\
\text { Frequency } \\
(\mathrm{n}=746)\end{array}$ & $\begin{array}{c}\text { Percentage of } \\
\text { Worsened Patients } \\
\text { (frequency) }\end{array}$ & RR: OR (95\% CI) \\
\hline 0 & 224 & $7.6 \%$ & $0.13(0.08-0.21)$ \\
\hline 1 & 129 & $13.9 \%$ & $0.33(0.19-0.55)$ \\
\hline 2 & 59 & $20.3 \%$ & $0.58(0.30-1.24)$ \\
\hline 3 & 169 & $44.4 \%$ & $2.31(1.62-3.30)$ \\
\hline 4 & 116 & $58.6 \%$ & $4.31(2.88-6.55)$ \\
\hline 5 & 29 & $72.4 \%$ & $6.69(2.92-15.35)$ \\
\hline $6-8$ & 20 & $60 \%$ & $3.66(1.47-9.08)$ \\
\hline
\end{tabular}

\section{Case Examples \\ Case 1: Clival Chordoma With Milan Complexity Scale Score 6}

A 37-year-old female patient came to our attention due to 4th cranial nerve palsy, dysphagia, gait imbalance, and left lower-limb weakness. The patient's preoperative KPS score was 70 . The preoperative brain MR image showed a $3 \times 3 \times 3.5-\mathrm{cm}$ lesion arising from the clivus and developing posteriorly and superiorly, causing brainstem compression and secondary hydrocephalus (Fig. 1). Her Milan Complexity Scale score was 6 (eloquent area involved, 3 points; cranial nerve manipulation, 2 points; posterior fossa surgery, 1 point; no major brain vessel manipulation and tumor size $<4.1 \mathrm{~cm}, 0$ points). She underwent total tumor removal (histopathology: clival chordoma) followed by proton beam radiotherapy. All of her symptoms improved, apart from dysphagia which slightly worsened and required temporary tracheostomy. At discharge, her KPS score was 70 .

\section{Case 2: Pinealoblastoma With Milan Complexity Scale Score 5}

A 56-year-old female patient's neurological history began when she presented with progressive ataxia and gait impairment. Brain MRI with intravenous contrast administration depicted a $3 \times 3 \times 3-\mathrm{cm}$ pineal region tumor with
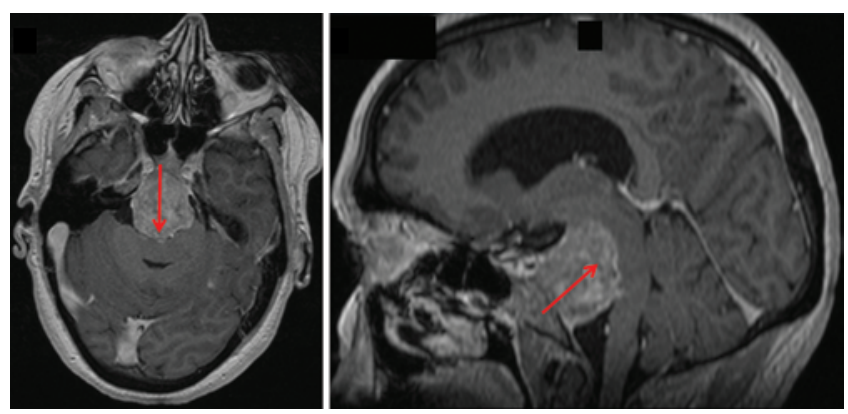

FIG. 1. Case 1. Preoperative axial (left) and sagittal (right) brain T1weighted MR images with intravenous contrast administration (clival chordoma). The red arrows indicate the brainstem compression. The Milan Complexity scale score was 6: eloquent area (brainstem), 3 points; multiple cranial nerve manipulation during surgery (cranial nerves III, IV, V, VII, and VIII), 2 points; posterior fossa surgery, 1 point. intense contrast enhancement associated with aqueductal compression and secondary hydrocephalus. There was encasement of the deep venous system (Fig. 2), and the Milan Complexity Scale score was 5 (eloquent area involved, 3 points; major brain vessel involvement, 1 point; posterior fossa surgery, 1 point; no manipulation of the cranial nerves and tumor size $<4.1 \mathrm{~cm}, 0$ points). When she was admitted to the hospital, her neurological examination revealed severe gait imbalance (not able to walk on her own) and a positive Romberg sign with marked dysmetria at the upper limbs (left more than right) with slight hypertonia of the lower limbs. Her KPS score was 60. She underwent STR (histopathology: pinealoblastoma), but her postoperative course was characterized by acute cerebellar swelling that required posterior fossa decompressive craniectomy, ventriculoperitoneal shunt, and tracheostomy. At discharge, her KPS was 40.

\section{Case 3: Left Rolandic Glioblastoma With Milan Complexity Scale Score 3}

A 77-year-old male patient was referred to our center due to a left Rolandic mass causing slight speech impairment and right upper-limb weakness. His preoperative KPS score was 80 . Preoperative brain MR with intravenous contrast administration showed a $3 \times 3.5 \times 2.5-$ $\mathrm{cm}$ left Rolandic high-grade glioma (Fig. 3). His Milan Complexity Scale score was 3 (eloquent area involved, 3 points; no major brain vessel or cranial nerve manipulation, no posterior fossa surgery, and tumor size $<4.1 \mathrm{~cm}$, 0 points). He successfully underwent complete tumor removal (histopathology: glioblastoma) with intraoperative motor function monitoring and was discharged home with improvement of his preoperative deficits (KPS Score 90).

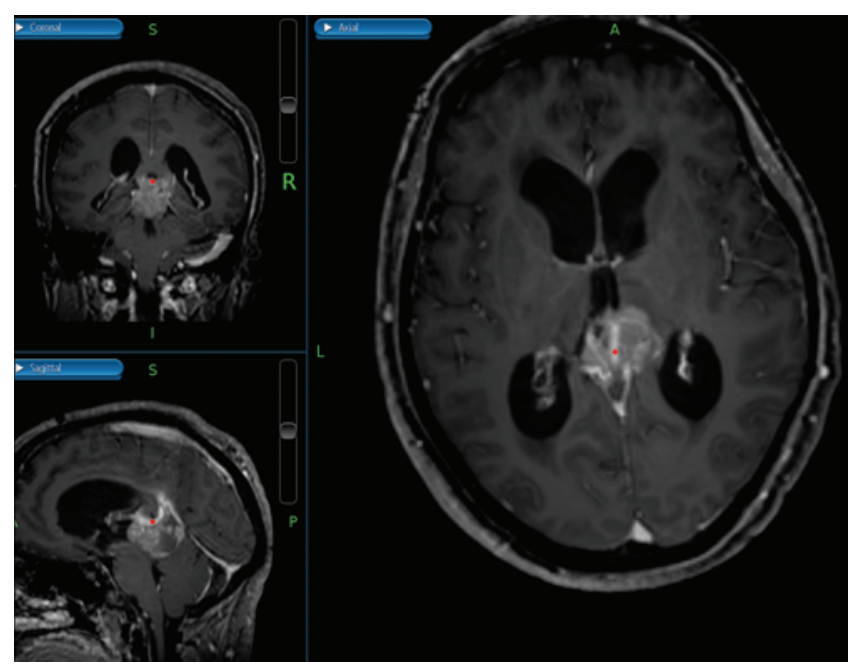

FIG. 2. Case 2. Images taken from the neuronavigation system (StealthStation S7, Medtronic) (pinealoblastoma) showing coronal, sagittal, and axial T1-weighted MR images with intravenous contrast administration. There is a $3 \times 3 \times 3-\mathrm{cm}$ strongly enhancing pineal region lesion with aqueductal compression, secondary hydrocephalus, and encasement of the deep venous system (red dots). The Milan Complexity scale score was 5: eloquent area (pineal region), 3 points; major brain vessel manipulation (internal cerebral veins; red dots), 1 point; posterior fossa surgery, 1 point. 


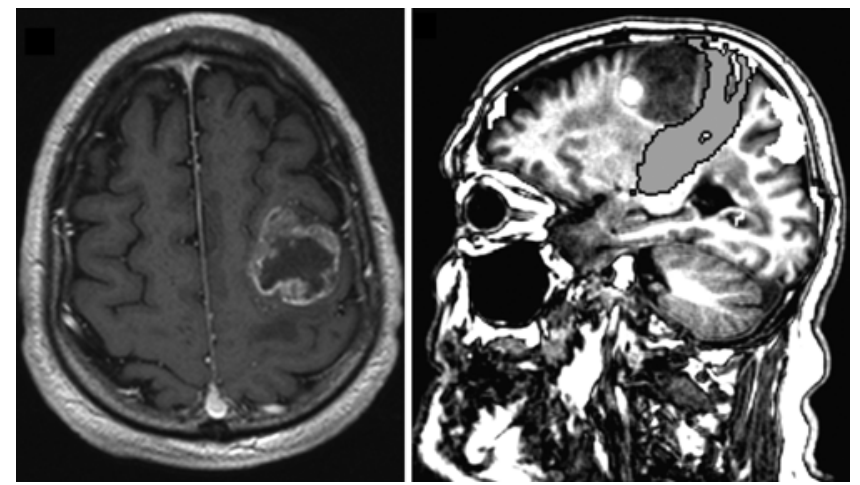

FIG. 3. Case 3. Preoperative axial brain T1-weighted MR image with intravenous contrast administration (left) and sagittal tractography MR image (right) (left Rolandic glioblastoma). The tractography shows the close relation between the corticospinal tract (gray) and the tumor. The Milan Complexity scale score was 3: eloquent area (motor region), 3 points.

\section{Case 4: Right Temporal Glioblastoma With Milan Complexity Scale Score 0}

A 74-year-old male patient underwent brain MRI because 2 months prior he started to complain of headache and confusion. This examination revealed a right posterior, temporal, $2.5 \times 2.5 \times 3-\mathrm{cm}$, round lesion suspected of being a high-grade glioma (Fig. 4). His preoperative KPS score was 90, and his Milan Complexity Scale score was 0 (no eloquent area, no manipulation of the major brain vessel or cranial nerves, no posterior fossa surgery, and tumor size $<4.1 \mathrm{~cm})$. He underwent surgery for complete tumor removal (histopathology: glioblastoma). After the operation, he did not show any new neurological deficits and he was discharged in good condition (his KPS score was 90).

\section{Discussion}

The dream of any patient affected by a brain tumor is to become free of his/her disease without having his/ her skull violated. Unfortunately, at the beginning of the 21 st century, we are still in the early stages of considering histological analysis and "maximal safe resection" as the gold standard for the treatment of the majority of brain tumors. ${ }^{1,27,32}$ For this reason, after having accepted the idea of undergoing surgery, patients spend a great amount of energy in a difficult selection process that is aimed to find the "best" place to receive treatment. If we, as surgeons, think about what should be the features of this best place, it is easy to identify the following aspects: 1) It must be a place where the surgeons are familiar with brain tumor surgery and advanced resection techniques. 2) It must be a place where valid indicators of efficacy are used, and the chance to have the tumor completely removed are maximized. 3) It must be a place where the risks of brain damage are quantified and minimized.

It is relatively easy to collect data regarding case volume, available technologies, and operative techniques, but it is very difficult to collect data regarding the indicators of efficacy and safety in terms of not only mortality, but also neurological outcome after surgery. The results of brain tumor surgery are, in fact, greatly influenced by case complexity, as shown by the results of our retrospective study
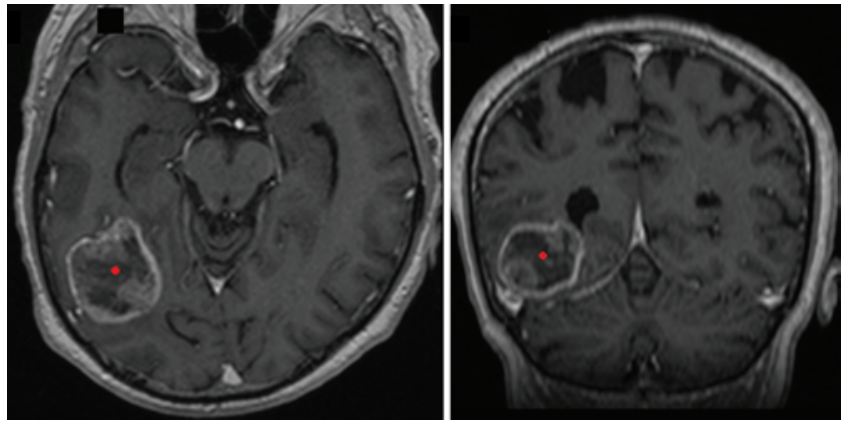

FIG. 4. Case 4. Preoperative axial (left) and coronal (right) T1-weighted brain MR images with intravenous contrast administration (right temporal glioblastoma; red dots). The Milan Complexity scale score was 0 in this case.

where 5 statistically significant predictors of the change in KPS scores were identified. The idea that case complexity in brain tumor surgery is related to the degree of resection and surgical mortality and morbidity is well-known and accepted in the neuroscientific community. ${ }^{9}$

This is the first known study to try to grade complexity by building a dedicated scale. The Milan Complexity Scale aims to provide a score that addresses the complexity of the neurosurgical procedure and translate the feeling of complexity that surgeons deal with while evaluating the relationships between the tumor and the surrounding structures on radiological images. This feeling of complexity is somewhat comparable to the feeling of complexity that a climber feels while looking at a mountain wall, which he/she is able to approximate into a universally accepted score that is able to convey this feeling to other climbers and has implications in training and the resources required for safe climbing. Despite the ability of experienced neurosurgeons to feel the complexity of a resection while looking at a patient's tumor, a common language that shares this feeling with colleagues is still lacking in neurooncological surgery. These complex factors that are so well described in the mountain-climbing world have been rarely or clearly identified in neurooncology.

What is it that makes craniotomy for tumor resection more likely to create neurological injury? The Milan Complexity Scale is able to estimate the risk of a negative clinical course in brain tumor surgery that indicates the worsening of health status at discharge. Its ability was also confirmed when secondary analyses were carried out on subsamples of cases with GTR and STR, as well as cases with glioma and meningioma histology: in all of these subgroups, the scores of the Milan Complexity Scale were significantly higher among those with a worsened KPS score in comparison with those with improved/unchanged KPS. This scale is composed of 5 items - the Big Fivenamely, major brain vessel manipulation, surgery in the posterior fossa, cranial nerve manipulation, surgery in an eloquent area, and tumor size. The scale's score ranges between 0 and 8 , and scores higher than 3 are indicative of an increased risk of worsening. Lower scores (approximately $0-2$ ) are easier to interpret and can be defined by relatively few variations in the scale's items, and the same can be said for the highest scores (i.e., 7-8). On the contrary, intermediate scores (i.e., those ranging from 4 to 6 ) should 
be carefully evaluated because the risk of worsening may differ according to the factors that define such scores.

The advantages of the Milan Complexity Scale include the following: it is easy to use, practical, intuitive, and quick. Therefore, the use of the Milan Complexity Scale is a viable way to predict the safety and outcome of neurosurgical procedures, and hence predict quality as well as appropriately adjust risk for programs that measure brain tumor quality. Moreover, we expect the Milan Complexity Scale will allow us to better inform patients on the likelihood that their postoperative clinical status will improve, worsen, or be stable on the basis of preoperative clinical conditions. Since the Big Five were found to be associated with worse outcome, it appears evident how patient selection can influence the indicators of outcome and how any attempt to compare neurosurgical team performance must account for these findings. In other words, despite the fact that it is evident to all neurosurgeons that the mortality in a clinical series of big posterior fossa tumors has a radically different meaning from mortality in a clinical series of small supratentorial tumors, these concepts are somewhat less evident to administrators and politicians and require numbers and data to be sustained.

Health care system financing in Italy is based on the average cost of care per patient as represented by the $\mathrm{Di}$ agnosis-Related Group, a classification system that identifies homogeneous groups of patients in terms of consumed resources. ${ }^{14}$ The concepts of the Case Mix Index (measure of case complexity) and the average weight of the Diagnosis-Related Group have been introduced by administrators, but they often lack the ability to identify the most severe patients. These indicators appear far from giving the right value to the Big Five, and any comparison among different brain tumor centers might be strongly biased by ignoring their importance. At present, the accreditation of neurosurgery centers in Italy is based on structural, organizational, and technological parameters such as the presence of a dedicated intensive care unit, instruments such as surgical microscopes, and experienced neurosurgeons and other qualified staff members. In some regions in Italy, case volume is another accreditation parameter for neurosurgery centers that is used as a criterion for safety and effectiveness. In our opinion, criteria such as case complexity and the postoperative worsening rate should be taken into account as well. A center should be accredited if its series is characterized by a rate of cases with postoperative worsening that is consistent with those observed in Italian centers with higher case volumes. Small centers should obtain the accreditation only if they have an experienced neurosurgeon on staff who is able to provide adequate postoperative results from a relevant personal series.

If the prognostic meaning of the Big Five will also be confirmed by others, the implications in education, training, resource allocation, and ethical and legal issues will become evident. As far as education and training are concerned, it is evident that tumor case complexity should be graded in order to plan the educational processes of a surgeon in a progressive "crescendo," where suddenly jumping from simple tumors to highly difficult cases should be avoided. ${ }^{34}$ In terms of resource allocation, it should appear clear to administrators that, by measuring case complexity in terms of the likelihood of postoperative deterioration, they will be able to grade centers on the basis of both case complexity and outcomes for patients. This new indicator might contribute to creating a meritocratic way to ensure that the best centers receive more attention and resources. Regarding ethical and legal issues, we all know that brain tumors comprise a variety of diseases with an incredible range of complexity and different outcomes. The Milan Complexity Scale could allow patients to be aware of the clinical factors they have that can influence surgical outcome before surgery, and therefore take conscious and informed decisions with the surgeon.

In this sense, the Milan Complexity Scale might help in the decision-making process in terms of the "operability" of a specific case and the evaluation of postoperative results and damage. This is still a topic where the patients receive a variety of different opinions ranging from "Do not touch: it is too dangerous," to "Ok, we know that the best chance to increase your life expectancy comes from gross-total resection. We collected a series of cases of tumors that are very similar to your case that we removed with an acceptable mortality and morbidity; thus, let's do it." ${ }^{37}$ In our series, more than $90 \%$ of the patients achieved complete or almost complete tumor removal, thus bringing attention to the fact that the relationship between case mix - defined as the ratio between complex and total cases-and indicators of efficacy (namely, the percentage of tumor resection across cases) is of paramount importance. Of course, these results need to be interpreted in the frame of a defined approach, i.e., maximal safe resection, and it is likewise clear that the experience and expertise of single neurosurgeons with particular diseases make this concept somewhat variable: younger and less-experienced neurosurgeons are more likely to undertake less aggressive approaches than those with more experience. However, if we take quality into account as a result of the safety and outcome of neurosurgical series from neurosurgical centers, where younger and older surgeons work together, the validity of the Milan Complexity Scale is expected to be confirmed. In our center, for example, neurosurgeons have on average 15 to 20 years of experience, which varies between 3 and 40 years among individual surgeons.

We recognize that the reported complication rate is fairly high $(41.7 \%)$ in the presented series, with almost $30 \%$ of KPS scores declining after surgery, but it should be underlined that most postoperative declines in the KPS scores were reversible. In this series, $28.9 \%$ of treated tumors were located in an eloquent area: these were all operated on with the aid of a combination of functional MR, tractography, intraoperative monitoring, and awake surgery, ${ }^{11}$ which allowed us to push resection very close to the eloquent tissue and often implies a temporary worsening of neurological functions. Indeed, postoperative KPS was calculated at hospital discharge, and most of these worse cases were represented by reversible deficits that then fully recovered either spontaneously or with physiotherapy.

Previous studies have proposed grading systems that are able to predict a patient's clinical outcome. Chelazzi et al. created a score to predict the occurrence of postoperative complications in general surgery. Similar to us, their aim was to identify high-risk surgical patients through a 
simple, economical, and objective scoring system. ${ }^{8}$ Lawton et al. developed a grading system to predict neurological outcomes after surgery for brain arteriovenous malformations with the aim of improving and refining patient selection. ${ }^{25}$ To the best of our knowledge, similar studies are lacking in neurooncological surgery, and the Milan Complexity Scale is the first scale to be created ad hoc for neurooncology. The classifications of complications were based on etiology and the required treatment ${ }^{23}$ and useful for data analysis. It was shown that the majority of complications were minor in the present series, and they did not require any invasive treatment. Regarding etiology, the fact that surgical manipulation of eloquent nervous tissue was by far the principal cause of complication was not a surprise. Indeed, this was somehow expected, as the purpose of our institution is to pursue, whenever possible, complete tumor removal according to the "maximal safe resection" concept. ${ }^{1,27,32}$

Our study has 3 main limitations. First, the scale was built using retrospective data and therefore should be validated in a new cohort of patients with brain tumors in order to prospectively evaluate the efficacy of the Milan Complexity Scale. Parallel to this, it has to be acknowledged that the approach to treatment was maximal safe resection. It has to be tested whether or not more aggressive approaches might yield similar performances on this scale. Second, our results should be cautiously generalized since this case series was based on patients attending a third-level referral center, where it is likely that surgical series are representative of the most severe situations. Third, we took into account only neurological variables and excluded other internal conditions (e.g., diabetes, heart disease). Future research should be performed, including research by different centers that takes into account other factors that can influence surgical outcome, e.g. surgical techniques and neurosurgeons' experience, but also nonneurological comorbidities that might influence clinical decisions regarding operability and postsurgical course. Moreover, future studies should consider long-term functional impairment, i.e., the patient's clinical situation at months after surgery.

\section{Conclusions}

By evaluating 5 parameters (major brain vessel manipulation, posterior fossa surgery, cranial nerve manipulation, eloquent area involvement, and tumor size $>4 \mathrm{~cm}$ ), the $\mathrm{Mi}$ lan Complexity Scale enables both the neurosurgeon and patient to estimate the risk of a negative clinical course after brain tumor surgery.

We expect that, in the future, the Milan Complexity Scale will be used for several purposes: clinical purposes (i.e., predicting the quality of brain tumor surgery in terms of safety and outcome), research (i.e., comparison between different centers' quality of treatment provided), education (i.e., referring residents and fellows to high-quality centers), and better health system management (i.e., quality measurement programs and resource allocation).

\section{Acknowledgments}

We thank the IEN Foundation for their support.
This research is dedicated to Dr. Robert Spetzler and celebrates the 3 months of observation that Dr. Paolo Ferroli spent in Phoenix in 2003. By his simple example, he planted in Dr. Ferroli the seeds of dedication to knowing surgical anatomy, surgical techniques, and the honest recognition and analysis of clinical results.

\section{References}

1. Almeida JP, Chaichana KL, Rincon-Torroella J, QuinonesHinojosa A: The value of extent of resection of glioblastomas: clinical evidence and current approach. Curr Neurol Neurosci Rep 15:517, 2015

2. Apuzzo MLJ: Brain Surgery. Complication Avoidance and Management. New York: Churchill Livingstone, 1993, Vol $1, \mathrm{p} \times x i$

3. Barker FG II, Curry WT Jr, Carter BS: Surgery for primary supratentorial brain tumors in the United States, 1988 to 2000: the effect of provider caseload and centralization of care. Neuro Oncol 7:49-63, 2005

4. Barker FG II, Klibanski A, Swearingen B: Transsphenoidal surgery for pituitary tumors in the United States, 1996-2000: mortality, morbidity, and the effects of hospital and surgeon volume. J Clin Endocrinol Metab 88:4709-4719, 2003

5. Behrens E, Schramm J, Zentner J, König R: Surgical and neurological complications in a series of 708 epilepsy surgery procedures. Neurosurgery 41:1-10, 1997

6. Black PM: What is a complication in neurosurgical surgery? A practical approach, in Apuzzo MLJ (ed): Brain Surgery. Complication Avoidance and Management. New York: Churchill Livingstone, 1993, Vol 1, pp xxv-xxvii

7. Bonsanto MM, Hamer J, Tronnier V, Kunze S: A complication conference for internal quality control at the Neurosurgical Department of the University of Heidelberg. Acta Neurochir Suppl (Wien) 78:139-145, 2001

8. Chelazzi C, Villa G, Vignale I, Falsini S, Boni L, De Gaudio AR: Implementation and preliminary validation of a new score that predicts post-operative complications. Acta Anaesthesiol Scand 59:609-618, 2015

9. Clark JC, Spetzler RF: Creating a Brave New World for neurosurgery. World Neurosurg 75:608-609, 2011

10. Clavien PA, Sanabria JR, Strasberg SM: Proposed classification of complications of surgery with examples of utility in cholecystectomy. Surgery 111:518-526, 1992

11. Cordella R, Acerbi F, Broggi M, Vailati D, Nazzi V, Schiariti $\mathrm{M}$, et al: Intraoperative neurophysiological monitoring of the cortico-spinal tract in image-guided mini-invasive neurosurgery. Clin Neurophysiol 124:1244-1254, 2013

12. Dasenbrock HH, Clarke MJ, Witham TF, Sciubba DM, Gokaslan ZL, Bydon A: The impact of provider volume on the outcomes after surgery for lumbar spinal stenosis. Neurosurgery 70:1346-1354, 2012

13. Davies JM, Yanamadala V, Lawton MT: Comparative effectiveness of treatments for cerebral arteriovenous malformations: trends in nationwide outcomes from 2000 to 2009. Neurosurg Focus 33(1):E11, 2012

14. Decreto legislativo 30 dicembre 1992 n. 502. Riordino della disciplina in materia sanitaria, a norma dell'articolo 1 della legge 23 ottobre 1992, n. 421

15. Dindo D, Demartines N, Clavien PA: Classification of surgical complications: a new proposal with evaluation in a cohort of 6336 patients and results of a survey. Ann Surg 240:205213, 2004

16. Emanuel E, Tanden N, Altman S, Armstrong S, Berwick D, de Brantes F, et al: A systemic approach to containing health care spending. N Engl J Med 367:949-954, 2012

17. Ferroli P, Brock S, Leonardi M, Schiavolin S, Acerbi F, Broggi M: Complications in neurosurgery: application of Landriel Ibañez classification and preliminary considerations on 1000 cases. World Neurosurg 82:e576-e577, 2014 
18. Ferroli P, Caldiroli D, Leonardi M, Broggi M: Complications in neurosurgery: the need for a common language. J Neurosurg 122:983-984, 2015 (Letter)

19. Glickman SW, Schulman KA, Peterson ED, Hocker MB, Cairns CB: Evidence-based perspectives on pay for performance and quality of patient care and outcomes in emergency medicine. Ann Emerg Med 51:622-631, 2008

20. Goslings JC, Gouma DJ: What is a surgical complication? World J Surg 32:952, 2008

21. Gough I: What is a surgical complication? World J Surg 32:950-951, 2008

22. Houkin K, Baba T, Minamida Y, Nonaka T, Koyanagi I, Iiboshi S: Quantitative analysis of adverse events in neurosurgery. Neurosurgery 65:587-594, 2009

23. Landriel Ibañez FA, Hem S, Ajler P, Vecchi E, Ciraolo C, Baccanelli M, et al: A new classification of complications in neurosurgery. World Neurosurg 75:709-715, 604-611, 2011

24. Latimer K, Pendleton C, Olivi A, Cohen-Gadol AA, Brem H, Quiñones-Hinojosa A: Harvey Cushing's open and thorough documentation of surgical mishaps at the dawn of neurologic surgery. Arch Surg 146:226-232, 2011

25. Lawton MT, Kim H, McCulloch CE, Mikhak B, Young WL: A supplementary grading scale for selecting patients with brain arteriovenous malformations for surgery. Neurosurgery 66:702-713, 2010

26. Lebude B, Yadla S, Albert T, Anderson DG, Harrop JS, Hilibrand A, et al: Defining "complications" in spine surgery: neurosurgery and orthopedic spine surgeons' survey. J Spinal Disord Tech 23:493-500, 2010

27. Marko NF, Weil RJ, Schroeder JL, Lang FF, Suki D, Sawaya RE: Extent of resection of glioblastoma revisited: personalized survival modeling facilitates more accurate survival prediction and supports a maximum-safe-resection approach to surgery. J Clin Oncol 32:774-782, 2014

28. Nelson AR: Pay-for-performance programs: ethical questions and unintended consequences. J Fam Pract 56 (10 Suppl A): $16 \mathrm{~A}-18 \mathrm{~A}, 2007$

29. Nuño M, Mukherjee D, Carico C, Elramsisy A, Veeravagu A, Black KL, et al: The effect of centralization of caseload for primary brain tumor surgeries: trends from 2001-2007. Acta Neurochir (Wien) 154:1343-1350, 2012

30. Reponen E, Tuominen H, Korja M: Evidence for the use of preoperative risk assessment scores in elective cranial neurosurgery: a systematic review of the literature. Anesth Analg 119:420-432, 2014

31. Rolston JD, Han SJ, Lau CY, Berger MS, Parsa AT: Frequency and predictors of complications in neurological surgery: national trends from 2006 to 2011. J Neurosurg 120:736745,2014

32. Sanai N, Polley MY, McDermott MW, Parsa AT, Berger MS: An extent of resection threshold for newly diagnosed glioblastomas. J Neurosurg 115:3-8, 2011
33. Smith ER, Butler WE, Barker FG II: Craniotomy for resection of pediatric brain tumors in the United States, 1988 to 2000: effects of provider caseloads and progressive centralization and specialization of care. Neurosurgery 54:553565,2004

34. Snyderman C, Kassam A, Carrau R, Mintz A, Gardner P, Prevedello DM: Acquisition of surgical skills for endonasal skull base surgery: a training program. Laryngoscope 117:699-705, 2007

35. Sokol DK, Wilson J: What is a surgical complication? World J Surg 32:942-944, 2008

36. Spetzler RF, Martin NA: A proposed grading system for arteriovenous malformations. J Neurosurg 65:476-483, 1986

37. Teo C, Broggi M: Surgical outcome of patients considered to have "inoperable" tumors by specialized pediatric neurooncological multidisciplinary teams. Childs Nerv Syst 26:1219-1225, 2010 (Erratum in Childs Nerv Syst 26:1833, 2010)

38. Wong GK, Poon WS: Complications as the end point for neurosurgical and neurointerventional procedures: the way forward. World Neurosurg 75:604-605, 2011

39. Woolhandler S, Ariely D, Himmelstein DU: Why pay for performance may be incompatible with quality improvement. BMJ 345:e5015, 2012

\section{Disclosures}

The authors report no conflict of interest concerning the materials or methods used in this study or the findings specified in this paper.

\section{Author Contributions}

Conception and design: M Broggi, Ferroli, Acerbi, Bettamio, Caldiroli, Leonardi, Franzini, G Broggi. Acquisition of data: M Broggi, Cusin, La Corte, Visintini. Analysis and interpretation of data: M Broggi, Schiavolin, La Corte, Raggi. Drafting the article: M Broggi, Ferroli, La Corte. Critically revising the article: M Broggi, Ferroli, Schiavolin, Acerbi, Bettamio, Caldiroli, La Corte, Leonardi, Raggi, Schiariti, Franzini, G Broggi. Reviewed submitted version of manuscript: all authors. Approved the final version of the manuscript on behalf of all authors: M Broggi. Statistical analysis: Schiavolin, Raggi. Administrative/technical/material support: La Corte. Study supervision: M Broggi, Ferroli.

\section{Correspondence}

Morgan Broggi, Department of Neurosurgery, Fondazione IRCCS Istituto Neurologico Carlo Besta, via Celoria 11, Milano 20133, Italy. email: morganbroggi@ hotmail.com. 